\title{
Use of Shore Hardness Tests for In-Process Properties Estimation/Monitoring of Silicone Rubbers
}

\author{
H. Zhao*, D. Allanson, X. J. Ren \\ School of Engineering, Technology and Maritime Operations, Liverpool John Moores University, Liverpool, UK \\ Email: ${ }^{*}$ materials05@yahoo.co.uk, X.J.Ren@ljmu.ac.uk
}

Received 30 March 2015; accepted 24 June 2015; published 1 July 2015

\begin{abstract}
Casting is an important rubber manufacturing process for both production and material developments. A quick and flexible way of testing the constitutive materials properties of rubber products is very important for optimising the processing parameters and quality control. In many cases, standard tests such as tensile or compression tests are time consuming and require a large volume of materials. This work reports some recent work in using a combined numerical and experimental approach to characterise the properties of rubber materials during a casting process. Durometer shore hardness is used to test silicone rubbers (as a model material) with different compositions on different moulding planes and the linear elastic property is estimated from the hardnesses. The predicted properties are systematically compared with the experimental tests on hard and soft silicone rubber samples with different compositions. The work shows that shore hardness can be used as an effective way to monitor the materials properties during amoulding process for process optimisation and quality control.
\end{abstract}

\section{Keywords}

Silicone Room Temperature Vulcanization (RTV), Shore Hardness, Elastic Properties, FE Modelling, Quality Control

\section{Introduction}

Silicone rubber is one of the most important synthetic rubbers, used for various applications due to its excellent properties, such as flexibility, chemical resistance, and thermal stability [1]. There are many different silicone rubber manufacturing process such as high temperature vulcanization (HTV), room temperature vulcanization (RTV), liquid silicone rubber mould manufacturing (LSR), injection moulding, etc. [2]. The most efficient and quick way for silicone rubber casting and moulding is room temperature vulcanization approach. Casting is also commonly used as a convenient way to develop new grades of materials or to establish optimum manufacturing parameters [3]. A quick and easy way to assess the effects of composition change or manufacturing process is

"Corresponding author.

How to cite this paper: Zhao, H., Allanson, D. and Ren, X.J. (2015) Use of Shore Hardness Tests for In-Process Properties Estimation/Monitoring of Silicone Rubbers. Journal of Materials Science and Chemical Engineering, 3, 142-147.

http://dx.doi.org/10.4236/msce.2015.37019 
very important for improving the in-process quality control. A potential approach is using indentation tests, in which the resistance of the material is determined by either the depth of penetration for a predefined load or the load to reach a predefined indentation depth. One significant advantage of the indentation method lies in its simplicity and minimum requirement on the samples preparation and instrumentation.

The most relevant indentation test for rubber material is shore hardness tests. The method involves indenting the specimen using a hardened steel indenter with a specific geometry and force, based on the chosen scale of measurements. A mathematical scale is used to convert the displacement data into hardness values within a range between 0 and 100. According to international standards, the sample thickness should be at least $6.0 \mathrm{~mm}$ or over [4]. The shore hardness test method allows for hardness measurement on rubber specimen using a specified standard indenter corresponding to several rubber hardness measurement scales (A, B, C, D, DO, O, OO, and M) [4] [5]. It is widely used to evaluate the properties of materials such as elastomers, vulcanized rubber, cellular, gel-like, and plastics [6]. Shore hardness method is a high efficient testing method used in quality control, material selections and so on [7] [8]. However, current approach is not able to estimate the constitutive material properties such as Young's modulus, which is important for material design and selections. In addition, the effects of potential anisotropic properties associated with manufacturing and sample thickness are also not very clear which is important for material testing and quality control of manufacturing process [9] [10]. All these issues need to be addressed.

In this paper, shore A and $\mathrm{OO}$ hardness are used to monitor the hardness of vacuum cast silicone rubbers on different planes. The Young's modulus is successfully estimated by shore hardness values. The data showed a good agreement from experimental data of uniaxial compression test. The potential use of the shore OO hardness combined with FE modelling in quality control in rubber production is discussed.

\section{Experimental Works and Results}

The samples are made through the silicone room temperature vulcanization (RTV) vacuum curing approach. The mould for the silicone room temperature vulcanization was made of wood as shown in Figure 1(a), the mass of the silicone rubber solvent and the curing agent was calculated to control the silicone solvent-curing agent mass ratio. The solvent and the curing agent were mixed slowly for $10-15$ minutes. The mixture is degassed before pouring into the mould, and then degased again before the curing process. Figure 1(b) and Figure 1(c) shows the two samples with different resin and hardener ratios. Figure 1(b) is a rubber sample with a ratio of 1:10, Figure 1(c) is a rubber sample of a ratio of 1:20. These two samples have different hardnesses.

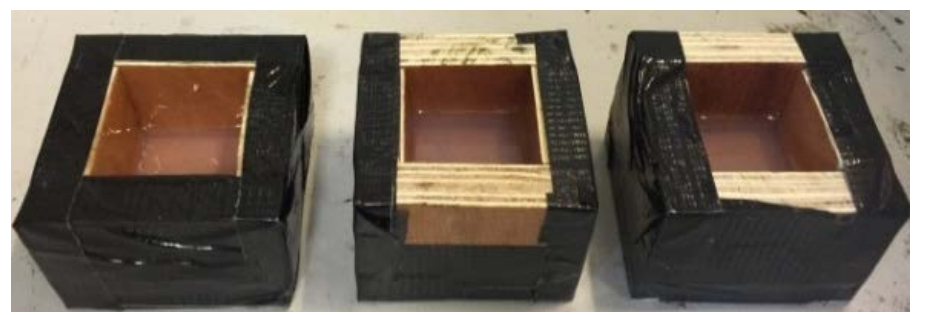

(a)

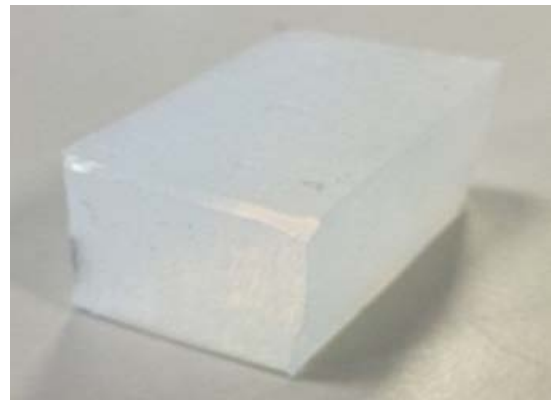

(b)

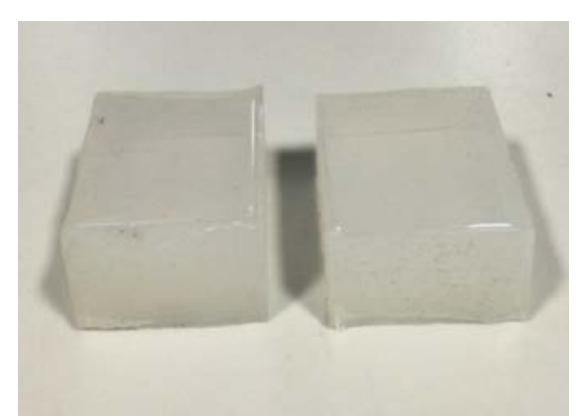

(c)

Figure 1. Mould used in the silicone rubber casting process and the samples with different mass ratios. (a) Mould used to cast silicone rubber sample in the lab; (b) Hard silicone rubber; (c) Soft silicone rubber. 
Two types of shore hardness have been assessed. One is shore A hardness and the other is shore OO hardness. The indenter shape and dimensions for the shore A and shore OO hardness tests are shown in Figure 2(a) \& Figure 2(b), respectively. During a measurement, a dead weight is used on the top of the Durometer indenter, 1 Kg for shore A hardness and $400 \mathrm{~g}$ for shore $\mathrm{OO}$ hardness for balancing the contact. The indentation load for shore A hardness test is $8.06 \mathrm{~N}$, the indentation load for the shore OO hardness test is $1.1 \mathrm{~N}$. The size of the moulding chamber is designed in a way that the hardness can be conveniently performed within the mould as shown in Figure 2(c) as a comparison to the tests performed when the sample is taken out of the mould.

\section{Standard Tests and FE Modelling}

To assess the potential anisotropy in the hardness of the sample, shore A and shore OO hardness tests have been performed on the different planes of the hard and soft silicone samples as schematically shown in Figure 3. This can be conveniently done with the shore A and shore OO hardness tester, which is a significant advantage. In each case, at least 6 tests are done the error bars represent the standard deviation of the six tests. As shown in Figure 3, the shore A hardness value is for the hard silicone rubber. It clearly shows that the hardness values with the sample in the mould or outside the mould is very close, the average value is close to 42 . The tests on the three planes are also in a good agreement, which confirms that the material can be treated as being isotropic. The shore OO hardness is for the soft rubber, again the hardness with the sample is in or outside the mould is comparable, and the hardness values taken on different planes are also very close. The Young's modulus is estimated from the shore hardness values based on a chart of the $S_{A}-t-E$ and $S_{O O}-t-E$ [11]. The estimated Young's modulus of the hard silicone rubber is 3.8MPa and the estimated Young's modulus of the soft silicone rubber is $1.6 \mathrm{MPa}$.

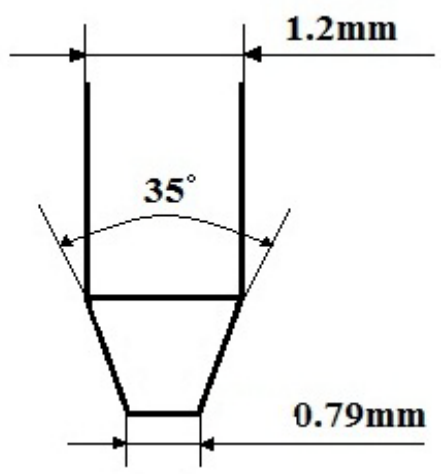

(a)

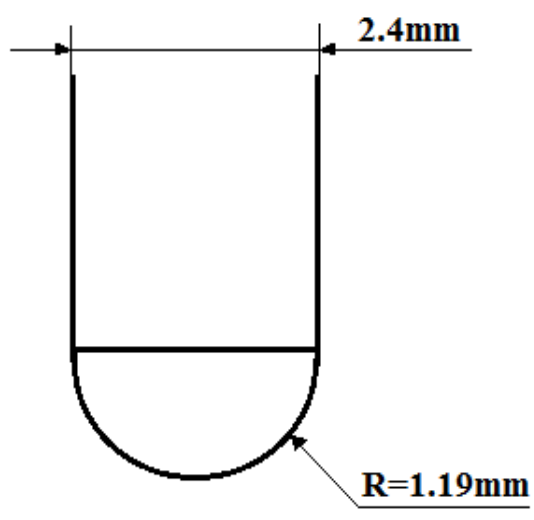

(b)

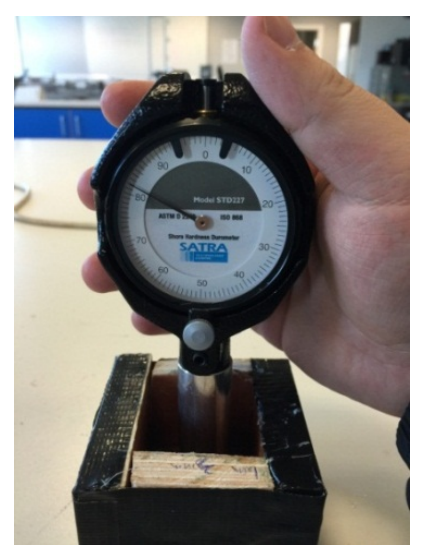

(c)

Figure 2. Schematic diagrams showing the shapes and dimensions of the indenters (a) \& (b) and the method to test the samples in the mould. (a) Shore A indenter tip and dimensions; (b) Shore OO indenter tip and dimensions; (c) Shore hardness test on the samples within the mould. 

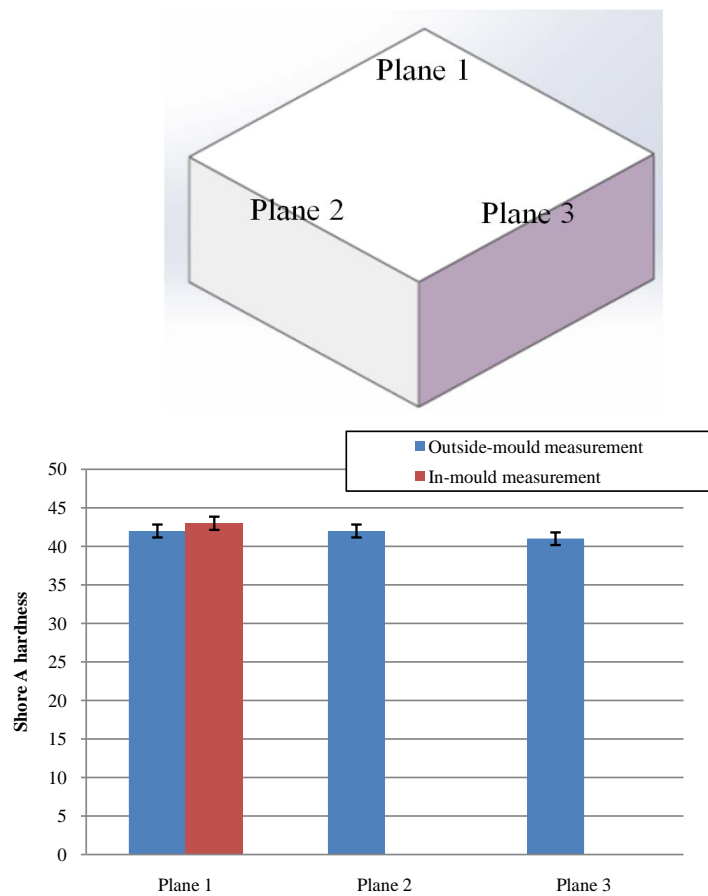

(a)
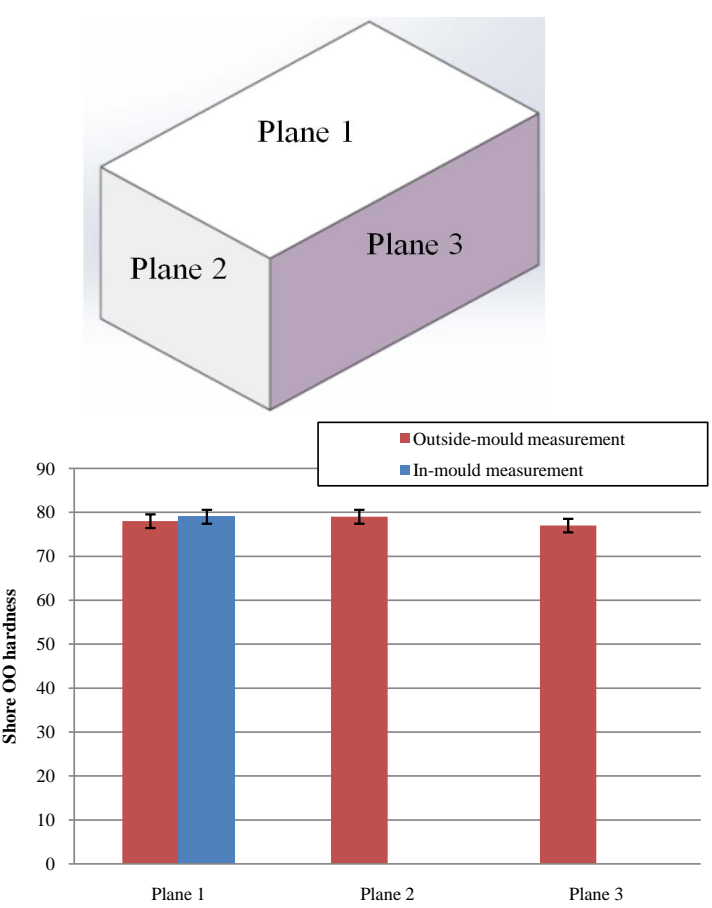

(b)

Figure 3. Shore hardness values tested with the sample is in the mould and outside the mould on three planes. (a) Shore A hardness of the hard silicone rubber sample; (b) Shore OO hardness of the soft silicone rubber sample.

To assess the accuracy of the estimated $\mathrm{E}$ values, compression tests have been performed on the rubber samples. The uniaxial compression test was performed on a tensile/compression machine (Figure 4(a)). Two flat plates were used to compress the sample, one flat plate was the support plate which is fixed at the bottom position, another flat plate was used to compress the sample vertically, which is attached to the load cell to record the force and the displacement. The compression test of the silicone rubber was simulated by a 3D FE model as shown in Figure 4(b). The element type used is 6-node linear triangular prism element type (C3D6), the width and thickness of the sample model is $40 \mathrm{~mm}$ and $20 \mathrm{~mm}$. In the FE model, the bottom plate is fixed by all degree of freedoms, and a displacement is applied to the top plate moving down onto the sample. A friction has been defined between the lower surface of the sample and the plate representing typical rubber metal friction [12]. The material properties used is the elastic modulus from the estimation based on the shore A hardness (Figure 3). Figure 5 shows the comparison of the experimental data and FE modelling results of the compression test. As shown in the figure, the FE predicted compression force displacement curve is in a reasonable agreement with the test data, which suggests that the E value predicted is reasonably accurate. Similar agreement could be found for both samples.

This work studied the properties of silicone rubber samples manufactured by different mass ratios of silicone solvent and curing agent through the room temperature vulcanization. The work showed that the different hardnesses of RTV silicone rubbers can be monitored by durometer shore hardness and the Young's modulus can be estimated from the shore hardness values, which is validated by standard compression test. This suggest that shore hardness could be routinely used to assess the properties in rubber manufacturing process to assess the property changes with varied compositions, different curing cycles and temperatures, different curing agent or particle reinforced used for enhancing mechanical properties of compositions [13] [14]. The prediction of E values from the shore hardness tests would further enhance the use of indentation method in quality control during rubber processing. A future direction of the work is to apply the same approach to other rubber systems and conditions.

\section{Conclusion}

In this paper, the use of shore hardness testing as a quick and convenient way of monitoring the properties of 


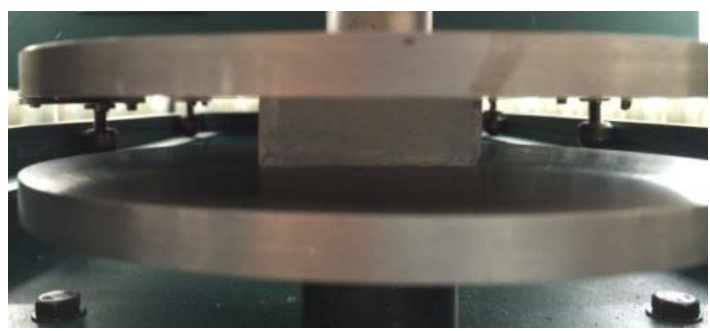

(a)

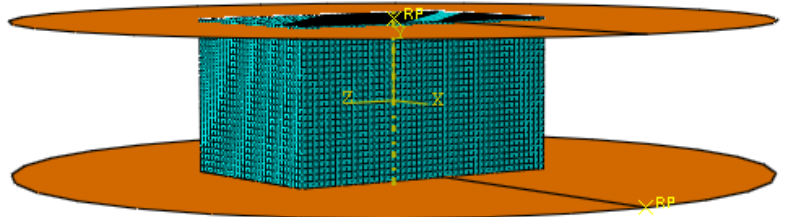

(b)
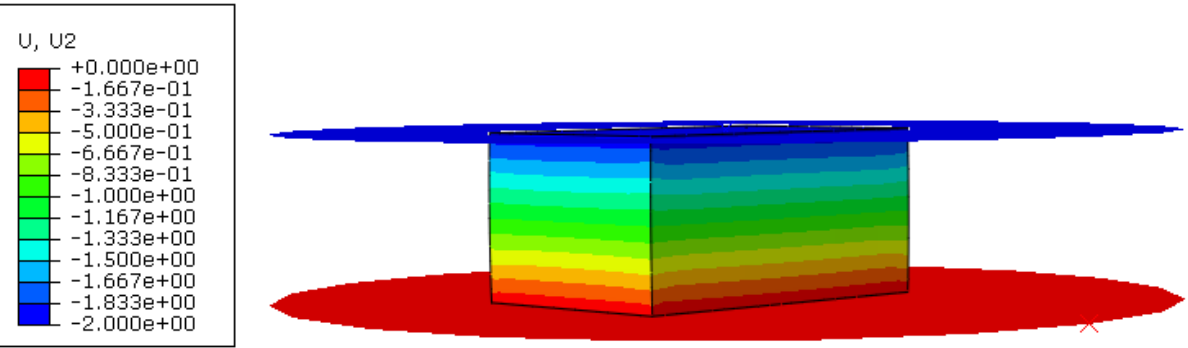

(c)

Figure 4. Compression tests and FE modelling. (a) Setup of the compression test on the soft silicone rubber sample; (b) FE model of the compression test; (c) Deformed shape (U2 is the vertical displacement).

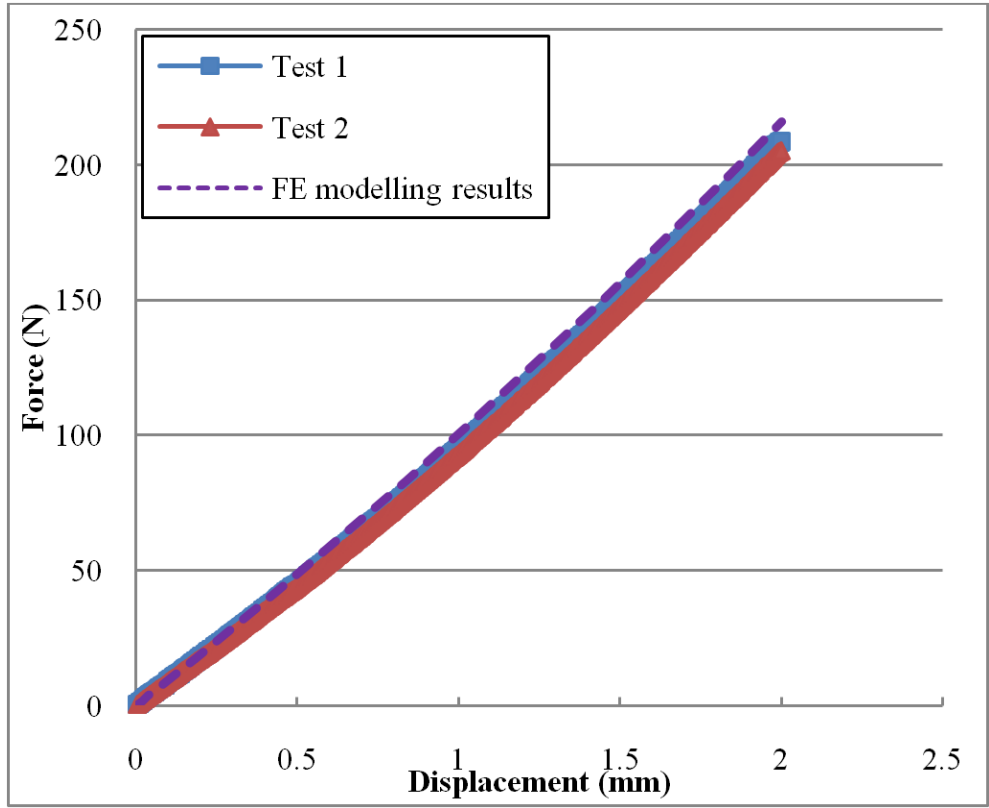

Figure 5 Comparison of the experimental force displacement curves of compression tests and FE prediction using the material properties inversely estimated based on the shore OO hardness tests.

silicone rubber during manufacturing is investigated. Two silicone rubber samples are manufactured with different mass ratios between silicone solvent and curing agent via the silicone room temperature vulcanization process. Systematic shore hardness tests have been performed on different moulding planes to evaluate potential anisotropy in the hardness. It is found that there is no significant hardness difference between different mould planes. The testing results with the sample in the mould and outside mould are also comparable. This potential could open us a way to monitor the in-mould material properties. Based on the shore hardness of the rubber samples, the corresponding Young's modulus is successfully estimated from the hardness values, which is vali- 
dated through comparison with tests and FE models of standard compression tests. This could further enhance the use of indentation tests in quality and property control in rubber manufacturing process.

\section{References}

[1] Kim, E.S., Kim, E.J., Lee, T.H. and Yoon, J.S. (2012) Clay Modification and Its Effect on the Physical Properties of Silicone Rubber/Clay Composites. Journal of Applied Polymer Science, 125, E298-E304. http://dx.doi.org/10.1002/app.36352

[2] Papailiou, K. and Schmuck, F. (2013) Material Selection and Manufacturing Processes for Composite Insulator with Silicone Rubber Housing. Silicone Composite Insulators Power Systems, 197-283. http://dx.doi.org/10.1007/978-3-642-15320-4_7

[3] Kalpakjian, S., Schmid, S.R. and Kok, C.W. (2008) Manufacturing Processes for Engineering Materials. PearsonPrentice Hall.

[4] ASTM D-2240 Standard Test Method for Rubber Property—Durometer Hardness.

[5] (2009) Durometer Shore Test Machine Handbook. INSTRON Ltd.

[6] (2011) Test Method for Measuring the Shore Hardness of Soft Materials Using the CETR-UMT 2 or CETR-APEX per ASTM D2240-00, Bruker Inc.

[7] Wakabayashi, K. (2011) Quality Control of Raw Materials. In: Handbook of Adhesion Technology, Springer, Berlin, 1007-1029. http://dx.doi.org/10.1007/978-3-642-01169-6_40

[8] Trifkovic, M., Sheikhzadeh, M., Choo, K. and Rohani, S. (2010) Experimental and Statistical Study of the Effects of Material Properties, Curing Agents, and Process Variables on the Production of Thermoplastic Vulcanizates. Journal of Applied Polymer Science, 118, 764-777. http://dx.doi.org/10.1002/app.32316

[9] Dinçkal, Ç. (2012) On the Properties of Anisotropic Engineering Materials Based upon Orthonormal Representations. IAENG International Journal of Applied Mathematics, 42, 3.

[10] Long, H. and Hamilton, S. (2008) Simulation of Effects of Material Deformation on Thickness Variation in Conventional Spinning. The 9th International Conference on Technology of Plasticity, South Korea.

[11] Zhao, H. (2015) Experimental and Numerical Modelling of Gasket Materials and Property Correlation. Ph.D. Thesis, Liverpool John Moores University, UK.

[12] Bensia, M., Bojan, B., Aleksandar, G. and Zoran, S. (2012) Computer-Aided Modelling of the Rubber-Pad Forming Process. Materials and Technology, 46, 503-510.

[13] Chen, D., Liu, Y. and Huang, C. (2012) Synergistic Effect between POSS and Fumed Silica on Thermal Stabilities and Mechanical Properties of Room Temperature Vulcanized (RTV) Silicone Rubbers. Polymer Degradation and Stability, 97, 308-315. http://dx.doi.org/10.1016/j.polymdegradstab.2011.12.016

[14] Zhang, J., Xu, Y.C. and Huang, P. (2009) Effect of Cure Cycle on Curing Process and Hardness for Epoxy Resin. Express Polymer Letters, 3, 534-541. http://dx.doi.org/10.3144/expresspolymlett.2009.67 